\title{
Sustained Virologic Response and Costs Associated with Direct-Acting Antivirals for Chronic Hepatitis C Infection in Oklahoma Medicaid
}

\author{
Timothy T. Pham, PharmD, PhD; Shellie L. Keast, PharmD, PhD; Kevin C. Farmer, RPh, PhD; \\ David M. Thompson, PhD, PT; R. Chris Rathbun, PharmD, BCPS, AQ-ID; Nancy J. Nesser, PharmD, JD: \\ Bethany P. Holderread, PharmD; and Grant H. Skrepnek, PhD, RPh
}

\begin{abstract}
BACKGROUND: Outcomes involving newer direct-acting antiviral (DAA) hepatitis $\mathrm{C}$ virus (HCV) regimens have not been studied extensively among the Medicaid population.

OBJECTIVE: To assess clinical (treatment failure) and economic outcomes for chronic HCV-infected Oklahoma Medicaid members following treatment with DAAs and to measure associations with patient, treatment, and clinical characteristics.

METHODS: This cross-sectional study used Oklahoma Medicaid pharmacy and medical claims data for adult members who used a newer DAA agent and had reported a successful or failed sustained virological response rate 12 weeks after therapy completion (SVR12) from January 1, 2014, to June 30, 2016. Multivariable logistic and gamma regressions assessed predictors of SVR12 failure and costs controlling for member demographics (i.e., age, sex, race, rural residence); type of DAA and adherence; clinical characteristics (e.g., comorbid conditions, advanced liver disease); and the implementation of changes to a prior authorization program.
\end{abstract}

RESULTS: Of 934 Medicaid members eligible for treatment with DAAs between January 1, 2014, and June 30, 2016, 906 received DAA treatment, $40.6 \%(368 / 906)$ had reported SVR12 outcomes, and 59.4\% $(n=538)$ did not have a reported SVR recorded. Of those with reported SVR12 outcomes, patients were $53.1 \pm 9.7$ years of age, $51.1 \%$ were male, $8.4 \%$ had SVR12 failure, and each member had mean costs of $\$ 140,283 \pm \$ 52,779$. Multivariable analyses indicated higher odds of SVR12 failure was independently associated with cirrhosis (OR [decompensated] $=6.69$ and $\mathrm{OR}$ [compensated] $=3.52, P<0.001$ ), while males had higher odds of failure than females ( $\mathrm{OR}=3.34, P<0.010)$. No significant difference in SVR12 failure was noted, according to DAA type or a medication adherence threshold of $>95 \%$. Ledipasvir/sofosbuvir was independently associated with lower costs $(\exp [b]=0.81 ; P<0.001)$ compared with sofosbuvir, while higher costs were associated with decompensated cirrhosis $(\exp [b]=1.22$; $P<0.001)$ and treatment failure $(\exp [b]=1.18, P<0.010)$. In an analysis including members without reported SVR12 outcomes, decompensated and compensated cirrhosis had lower odds $(P<0.001)$ of no reported SVR12 from ambulatory clinic settings.

CONCLUSIONS: Almost $60 \%$ of Medicaid members receiving DAA treatment did not have a final reported SVR12 outcome. Among those with viral load measurements, treatment success was high and both decompensated and compensated cirrhosis were independently associated with significantly higher odds of treatment failure. Addressing a loss to follow-up among HCV patients and curtailing the development of cirrhosis to improve treatment success may warrant interventions that improve access to care and remove barriers that impede treatment initiation and completion.

J Manag Care Spec Pharm. 2018;24(7):664-76

Copyright $\odot 2018$, Academy of Managed Care Pharmacy. All rights reserved.

\section{What is already known about this subject}

High-cost direct-acting antivirals (DAAs) for hepatitis C treatment present a formidable challenge to payers.

Public payers such as Medicaid cover the majority of beneficiaries chronically infected with hepatitis C.

Limited information exists on the management of hepatitis $C$ in Medicaid.

\section{What this study adds}

This study provides real-world evidence of clinical and cost outcomes with DAAs in the Medicaid setting.

The majority of Medicaid members who received DAA therapy did not have their final viral RNA tests reported for sustained virological response.

Cirrhosis of the liver was associated with failure to achieve sustained virological response.

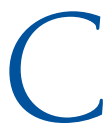

hronic hepatitis C virus (HCV) infection carries the risk of developing serious and debilitating liver sequelae and represents a high clinical and economic burden on the U.S. health care system. ${ }^{1-7}$ Between 1997 and 2007, an estimated 15,106 deaths were attributed to HCV, with higher risk of mortality associated with chronic liver disease, hepatitis B coinfection, alcohol-related conditions, minority status, and HIV coinfection. ${ }^{1,2}$ Progression from infection to severe illness varies widely, with $75 \%-85 \%$ of the infected population developing chronic infection, 60\%-70\% developing chronic liver disease, 5\%-20\% developing cirrhosis that may lead to hepatocellular carcinoma (HCC), and 1\%-5\% rapidly developing severe complications that result in death. ${ }^{3,4}$ Progression to serious liver disease is often asymptomatic until patients present with end-stage liver disease or HCC. ${ }^{4}$

According to the Centers for Disease Control and Prevention's National Health and Nutrition Examination Survey national data from 2003 to 2010, an estimated 3 million noninstitutionalized Americans were infected with chronic illness caused by $\mathrm{HCV}^{2}$ Greater risk of chronic infection was associated with males aged 40-49 years, of non-Hispanic black race, and with 
lower education levels and lower family income. The most common HCV transmission method is through percutaneous exposure to infected blood, including injection drug use, blood transfusion before the advent of anti-HCV tests in 1992, organ transplantation, needlestick injuries in health care settings, and birth to an HCV-infected mother. ${ }^{3}$

Treatment success for HCV infection is defined as sustained virological response (SVR) or an undetectable HCV RNA at 12 weeks after treatment (i.e., SVR12). ${ }^{8}$ Numerous treatment options for HCV have recently been introduced to the market. Before the advent of the direct-acting antivirals (DAAs), combination pegylated interferon and ribavirin were a mainstay of treatment, which achieved SVR in 54\%-61\% of patients but also high withdrawal rates due to debilitating adverse reactions. ${ }^{9}$ Two DAA protease inhibitors, boceprevir and telaprevir, were introduced in 2011 to treat HCV genotype 1 infection, ${ }^{10,11}$ with SVR rates of 66\%-68\% for boceprevir and 69\%-75\% for telaprevir. ${ }^{12}$ Despite higher SVR rates, these medications had relatively higher rates of adverse effects, including anemia and neutropenia. ${ }^{13}$ In 2013, newer second-generation DAAs were introduced with substantially higher SVR rates (i.e., >90\%) and fewer adverse events than older DAAs. ${ }^{14,15}$ Additional agents have been approved since then. ${ }^{16,17}$

The economic burden of HCV to the patient and to payers is substantial. A systematic literature review estimated the direct and indirect costs to payers of liver transplants at $\$ 201,110$, HCC at $\$ 23,766-\$ 44,200$, variceal hemorrhage at $\$ 25,595$, compensated cirrhosis at $\$ 585-\$ 1,110$, refractory ascites at $\$ 24,755$, hepatic encephalopathy at $\$ 16,430$, sensitive ascites at $\$ 2,450$, moderate chronic HCV at $\$ 155$, and mild chronic $\mathrm{HCV}$ at $\$ 145$ per year per person. ${ }^{5}$ An analysis of national discharges from the Agency for Healthcare Research and Quality Nationwide Inpatient Sample estimated that charges for hospitalizations with HCV increased from $\$ 0.9$ billion in 2004-2005 to $\$ 3.5$ billion during 2010-2011. ${ }^{6}$

Payer types for patients hospitalized for HCV were primarily Medicare and Medicaid, with an increasing trend for Medicare. ${ }^{6}$ Research evaluating health care utilization for HCV infection found $>2.3$ million outpatient, 73,000 emergency department (ED), and 475,000 inpatient visits annually in the United States from 2001 to 2010. ${ }^{7}$ The cost was greater than $\$ 15$ billion annually, and most visits were covered by Medicare and Medicaid. ${ }^{7}$

Newer DAAs present a major challenge to payers, especially public ones. Recent state Medicaid strategies to treat HCV, some of which have provoked much debate, include limiting access to patients with advanced liver disease (e.g., fibrosis stage 3 and 4, cirrhosis) and restricting access based on past alcohol and injection drug use. ${ }^{18}$ As public payers are under pressure to more equitably manage $\mathrm{HCV}$, real-world examples of HCV management and its outcomes become valuable to guide patient, payer, and provider decision making.
The purpose of this study, which focused on beneficiaries within a state Medicaid program, was to determine the clinical and economic outcomes for persons with chronic HCV infection following treatment and to assess the association between these outcomes and demographics, clinical characteristics, and DAA-related medication use from January 1, 2014, to June 30, 2016. Specific analyses also involved assessments of HCVrelated liver sequelae, medication adherence, and SVR12.

\section{Methods}

This investigation of a historical cohort used comprehensive, patient-level medical and pharmacy claims data from an administrative claims database provided by the Oklahoma Health Care Authority (OHCA) from January 1, 2014, to June 30, 2016, to Pharmacy Management Consultants (PMC)..$^{18-20}$ PMC, maintained by the University of Oklahoma College of Pharmacy, provides operational, consultant, and educational services to support OHCA in pharmacy benefit administration. The administrative data (i.e., claims) included information concerning medication use (e.g., type, quantity filled, days supply, date of service, prescriber, reimbursement amount); member demographics (e.g., age, sex, race, and residence type); and medical services (e.g., diagnoses, inpatient/outpatient/ED visits, dates of service, procedures, physician identifier, reimbursement amount).

These data were merged with additional clinical information reported by providers as required by OHCA's HCV prior authorization (PA) program. A PMC pharmacist oversees the PA process and maintains the database containing the clinical information for the HCV program. Throughout the course of therapy, providers report treatment regimen, initial viral load, treatment completion or discontinuation, and SVR12 outcomes. Oklahoma Medicaid initiated amendments to the PA program in July 2014 concerning DAA HCV medications, which included collection of additional information about treatment length, genotype, prior treatments, and fibrosis score. Notably, this revised PA program also implemented stricter treatment criteria, including a requirement for a fibrosis score of $\geq \mathrm{F} 2$ and no alcohol abuse or unauthorized injection drug use in the past 6 months. The Office of Human Research Participant Protection (institutional review board) at the University of Oklahoma Health Sciences Center and OHCA granted approval to this study as exempt.

Members identified for the study consisted of adults aged $\geq 18$ years who had (a) at least $1 \mathrm{HCV}$ diagnosis, including chronic or acute cases (International Classification of Diseases, Ninth Revision, Clinical Modification [ICD-9-CM]: 070.41, 070.51, 070.44, 070.54, 070.70, 070.71, V02.62; International Classification of Diseases, Tenth Revision, Clinical Modification [ICD-10-CM]: B17.10, B17.11, B18.2, B19.20, B19.21); (b) had initiated at least 1 DAA-containing regimen (sofosbuvir, simeprevir, sofosbuvir and simeprevir, 
ledipasvir/sofosbuvir, daclatasvir, or dasabuvir/ombitasvir/ paritaprevir and ritonavir) during the study period, and (c) reported a successful or failed SVR12. A successful SVR12 was defined as an undetectable HCV RNA at 12 weeks after treatment as reported by the provider, whereas a failed SVR 12 was defined as detected HCV RNA at 12 weeks.

During the course of the study, the large number of unreported SVR12s prompted further investigation. These members were analyzed as an additional subgroup. Members receiving telaprevir, boceprevir, or interferon-based regimens (i.e., interferon and ribavirin without a DAA) were excluded. Pharmacy and medical costs were compared between baseline and followup periods. These periods were demarcated by an index date, which was defined as the date of DAA initiation (i.e., the first date of service on administrative pharmacy claims). The baseline period included the 12-month pre-index date, and the follow-up period included the 12-month post-index date.

Outcomes of this study included SVR12 failure and total direct per member (PM) and per member per month (PMPM) pharmacy and medical costs. Costs were defined as the total amounts reimbursed by Medicaid as recorded in administrative claims. Only costs from the Medicaid database were available. Costs were inflation-adjusted to 2017 U.S. dollars using the prescription drugs and medical care services components of the Consumer Price Index (Appendix A, available in online article).

Independent variables assessed were based on prior research concerning SVR12 and direct medical costs, ${ }^{1-7,19,20}$ focusing primarily on validated theoretical frameworks regarding health service utilization..$^{21,22}$ As such, predictors included socioeconomic demographics (age, sex, race, rural or metropolitan residence); Deyo-Charlson comorbidity score for risk adjustment; comorbidities (e.g., cirrhosis, coinfection with HIV); medication use and adherence (to include treatment type and resource utilization of ED visits and hospitalizations); and HCV disease characteristics (e.g., viral load and fibrosis scores).

Following the amendments to the PA program on July 1, 2014, a treatment gap of $>3$ days per month was considered a criterion for subsequent denial of therapy and was therefore included as a predictor variable. The calculation for the treatment gap was based on the regimen's specific treatment length and the length of time in days between fill dates plus days supply. Members with a length of time between fill dates plus days supply $>3$ days were considered to have a gap. Members were determined to have discontinued treatment early if they did not have a days supply sufficient to fill their specific regimen's treatment length. Treatment length was either imputed or taken from their PA forms. Treatment lengths for members who initiated treatment before PA program amendments were imputed based on American Association for the Study of Liver Diseases-Infectious Diseases Society of America guidelines for treatment length during the preamendment period (12 or 24 weeks) and length of time between index date and last fill date plus days supply.
If administrative pharmacy claims had a combined days supply showing $\leq 12$ weeks of therapy, then 12 weeks was assigned as the length. If the combined days supply was $>12$ weeks, then 24 weeks was assigned. In this time period, genotype, fibrosis score, and treatment experience were not gathered on the PA form, precluding any clinical assessment.

To determine adherence for each member, the medication possession ratio (MPR) was calculated as the combined days supply from DAA therapy divided by the assigned treatment length in days. In general, MPR is expressed as a ratio of the days of the medication supply to the number of days in an assigned time interval, yielding a percentage value. ${ }^{23}$ Any MPR $>1$ was truncated to a value of 1 . An investigator manually determined combined days supply for a single course by summing all days supply for claims from and including the index date to probable end of therapy. Any new DAA claim with a date of service conceivably after the full length of therapy (i.e., gap from last claim $>120$ days) was considered a restart. Restarts were only included in descriptive analysis.

Comorbidities were defined according to ICD-9-CM and ICD-10-CM codes. They included the conditions in the DeyoCharlson Comorbidity Index and the following conditions for liver sequelae:13,24 alcoholic cirrhosis, chronic hepatitis, nonalcoholic cirrhosis, biliary cirrhosis, hepatocellular carcinoma, esophageal varices, hepatic encephalopathy, portal hypertension, hepatorenal syndrome, other sequelae of chronic liver disease, gastrointestinal bleed, ascites, and liver transplant (Appendix B, available in online article). ${ }^{6,25,26}$ As clinical cirrhosis measures were not available in the database, ICD codes were used to create proxy definitions. Mild or compensated cirrhosis was defined as alcoholic, nonalcoholic, or biliary cirrhosis without advanced liver sequelae. Decompensated cirrhosis was defined as cirrhosis with advanced liver sequelae.

Descriptive statistics analyzed member, disease, and economic characteristics. Patients who experienced treatment success versus treatment failure and those who reported an SVR12 result versus those who did not were compared using Wilcoxon-Mann-Whitney tests for continuous variables, Pearson chi-square tests for categorical variables, and Fisher's exact test for categorical variables with small cell counts. Differences in means between patients' paired (and therefore dependent) pre-index and post-index utilization and costs were compared using Wilcoxon signed rank sum tests. Logistic regression with a Huber-White robust standard error estimation was used to model odds ratios (ORs) and 95\% confidence intervals (CIs) for SVR12 failure between groups while adjusting for important covariates. An additional logistic regression was conducted to model estimates for SVR12 nonreport. Loglinked gamma regression with Huber-White robust standard errors was used to compare groups on the ratios of total postinitiation expenditures. ${ }^{27}$ Regression diagnostics (e.g., deviance, residuals) were conducted to identify influence and 


\section{FIGURE 1 \\ Sample Selection Criteria for Members Who Received DAA Treatment and Reported SVR12}

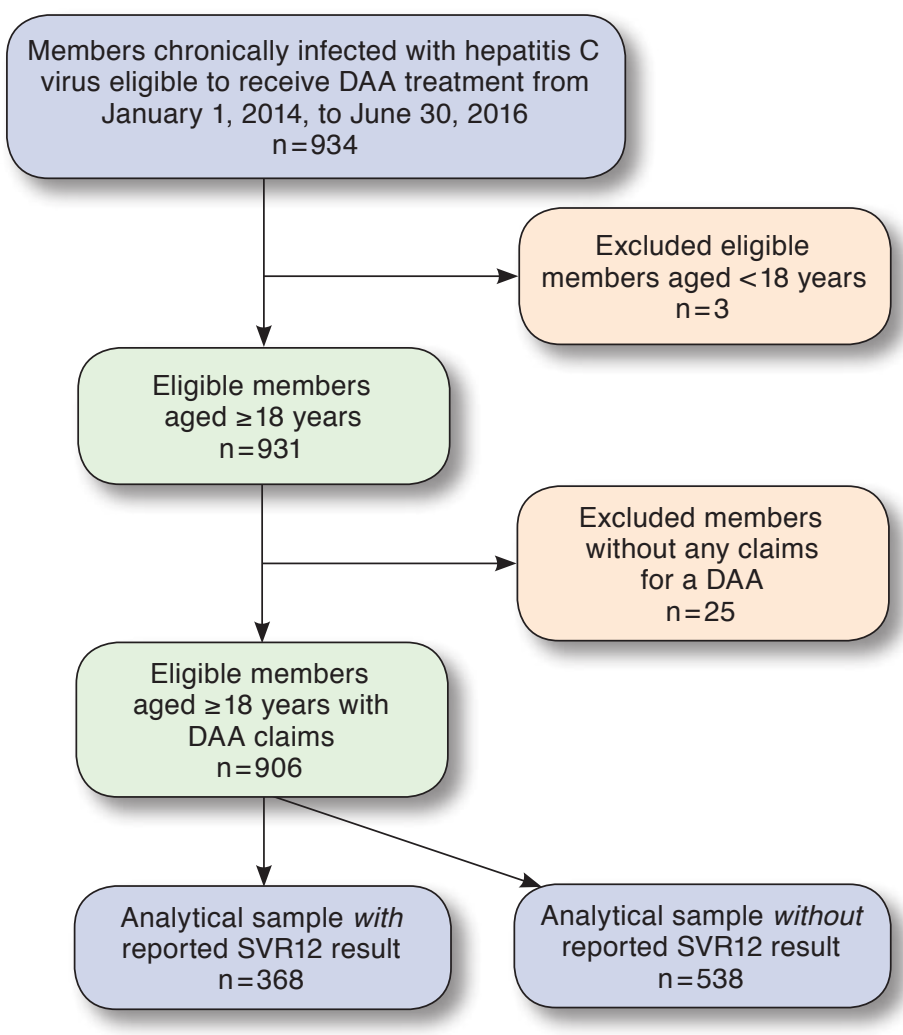

DAA = direct-acting antiviral; SVR12 = sustained virological response at 12 weeks

to assess model fit. Sensitivity analyses were conducted using alternate definitions of cirrhosis (e.g., dichotomized yes/no cirrhosis variable; decompensated cirrhosis defined as any presence of advanced liver disease with or without cirrhosis diagnoses) and adherence (MPR thresholds $\geq 0.80, \geq 0.95$, and 1.00) in order to assess the robustness and determine the sources of uncertainty in the models. ${ }^{28}$ All analyses were conducted with SAS software package 9.3 (SAS Institute, Cary, NC) and STATA MP 14.2 statistical software (StataCorp, College Station, TX). An alpha level of 5\% was considered for statistical significance.

\section{Results}

\section{Descriptive Statistics}

Of the 934 total Medicaid members who petitioned OHCA for HCV treatment, 906 had at least 1 claim for a DAA. Of the latter, 538 did not have any reported result for SVR12. Thus, 368 members met all inclusion criteria and had a reported HCV RNA/SVR12 result (Figure 1). At initiation of treatment, these 368 members averaged $53.1 \pm 9.7$ years of age, were $51.1 \%$ male, consisted of $77.7 \%$ self-identified white race, and $64.4 \%$ resided in metropolitan areas. Regarding treatment characteristics, $45.9 \%$ received sofosbuvir, $43.8 \%$ received the ledipasvir/ sofosbuvir combination, and $10.4 \%$ received differing regimens (e.g., daclatasvir, dasabuvir/ombitasvir/paritaprevir and ritonavir, and simeprevir). Most of the included sample of 368 had 12 weeks of treatment (73\%), followed by 24 weeks (16.0\%), 8 weeks (10.3\%), and 16 weeks (1.1\%). The mean DeyoCharlson Comorbidity Index score was $3.2 \pm 2.8$; treatment failures had higher index scores $(4.5 \pm 2.5$ vs. $3.1 \pm 2.8$ for SVR successes, $P=0.001)$. Treatment failures also reported significantly higher $(P<0.050)$ frequencies of alcoholic cirrhosis, nonalcoholic cirrhosis, portal hypertension, ascites, and decompensated cirrhosis. Overall MPR was high, at $0.98 \pm 0.10$, with no significant difference between successes and failures. Full descriptive statistics are presented in Table 1.

Also presented within Table 1, characteristics of the 538 members without an SVR12 reported indicated that nonreporters had fewer mild liver sequelae diagnoses (43.1\% vs. $52.7 \%, P<0.010$ ), fewer moderate to severe liver sequelae (24.5\% vs. $31.5 \%, P<0.050)$, and less cirrhosis $(34.0 \%$ vs. $44.3 \%, P<0.010)$, mainly driven by decompensated cirrhosis (18.0\% vs. $27.2 \%, P<0.010)$. Additionally, nonreporters had higher proportions of early treatment discontinuation $(18.8 \%$ vs. $6.0 \%, P<0.001)$.

Changes in utilization and costs for the 368 members from pre- to post-index are presented in Table 2. No differences were seen with inpatient hospitalizations or ED visits. Physician office visits increased for members with SVR12 failures $(10.9 \pm 5.6$ visits PM pre-index to $13.1 \pm 6.6$ visits PM post-index). As expected, due to increased medication use, overall expenditures increased $(\$ 19,872 \pm \$ 28,442$ PM preindex to $\$ 140,283 \pm \$ 52,779$ PM post-index, $P<0.001)$. HCV treatment cost increased from $\$ 1,156 \pm \$ 8,134$ PM before DAA use to $\$ 122,283 \pm \$ 46,819$ PM following use $(P<0.001)$. Medical expenditures decreased slightly $(\$ 14,015 \pm \$ 23,470$ PM pre-index to $\$ 12,327 \pm \$ 19,479$ PM post-index, $P<0.001$ ), potentially driven by decreases among SVR12 successes $(\$ 14,062 \pm \$ 24,174$ PM pre-index to $\$ 11,664 \pm \$ 17,990$ PM postindex, $P<0.001$ ).

Overall, 337/368 (91.6\%) members achieved SVR12 with median total costs PM of $\$ 119,423$ (interquartile range [IQR] $\$ 108,172-\$ 177,905)$. Women achieved 95.6\% (172/180) SVR12 with median costs of $\$ 117,642$ (IQR $\$ 106,505-\$ 177,905$ ) PM, and men achieved $87.8 \%$ (165/188) SVR12 with median costs of $\$ 121,004$ (IQR $\$ 109,535-\$ 176,114$ ) PM. Cases with cirrhosis had an SVR12 of 85.3\% (139/163), while noncirrhotic members achieved an SVR12 of 96.6\% (198/205). Median costs for cirrhosis were $\$ 135,748$ (IQR $\$ 114,829$-\$198,988) PM while those with no cirrhosis had median costs of $\$ 114,113$ (IQR $\$ 103,917$ $\$ 130,652)$ PM. 


\section{Sustained Virologic Response and Costs Associated with Direct-Acting Antivirals for Chronic Hepatitis C Infection in Oklahoma Medicaid}

TABLE 1 Baseline and Clinical Characteristics of Oklahoma Medicaid Members Receiving DAA Treatment and Evaluated for SVR12

\begin{tabular}{|c|c|c|c|c|}
\hline \multicolumn{5}{|l|}{ Member characteristics } \\
\hline Age, years, mean (SD) & $54.2(9.1)$ & $53.0(9.7)$ & $53.1(9.7)$ & $51.6(9.9)^{\mathrm{a}}$ \\
\hline \multicolumn{5}{|l|}{ Sex, $\%$} \\
\hline Male & $49.0^{\mathrm{b}}$ & $74.2^{\mathrm{b}}$ & 51.1 & 46.5 \\
\hline \multicolumn{5}{|l|}{ Race, \% } \\
\hline White & 77.5 & 80.7 & 77.7 & 77.1 \\
\hline Black & 9.5 & 9.7 & 9.5 & 8.6 \\
\hline Other & 13.1 & 9.7 & 12.8 & 14.3 \\
\hline Rural/metropolitan residence, $\%$ & 36.2 & 29.0 & 35.6 & $42.6^{\mathrm{a}}$ \\
\hline \multicolumn{5}{|l|}{ Region, \% } \\
\hline Unknown & $\leq 1.0$ & $\leq 1.0$ & $\leq 1.0$ & $2.2^{\mathrm{b}}$ \\
\hline Northeast & 12.8 & 12.9 & 12.8 & $19.0^{\mathrm{b}}$ \\
\hline Northwest & 4.8 & 6.5 & 4.9 & $5.0^{\mathrm{b}}$ \\
\hline Southeast & 16.9 & 9.7 & 16.3 & $18.8^{\mathrm{b}}$ \\
\hline Southwest & 6.5 & 9.7 & 6.8 & $6.3^{b}$ \\
\hline Oklahoma City & 38.6 & 51.6 & 39.7 & $25.7^{\mathrm{b}}$ \\
\hline Tulsa & 19.9 & 9.7 & 19.0 & $23.1^{\mathrm{b}}$ \\
\hline \multicolumn{5}{|l|}{ Treatment characteristics, \% } \\
\hline Initiated treatment after amendments to the prior authorization program & 68.3 & 54.8 & 67.1 & 87.2 \\
\hline \multicolumn{5}{|l|}{ Regimen } \\
\hline Sofosbuvir & 45.1 & 54.8 & 45.9 & $22.5^{c}$ \\
\hline Ledipasvir/sofosbuvir & 44.8 & 32.3 & 43.8 & $53.2^{\mathrm{b}}$ \\
\hline Daclatasvir & 3.3 & $\leq 1.0$ & 3.0 & $3.7^{\mathrm{b}}$ \\
\hline Dasabuvir/ombitasvir/paritaprevir and ritonavir & 2.4 & $\leq 1.0$ & 2.2 & $1.5^{\mathrm{b}}$ \\
\hline Sofosbuvir/ledipasvir & NA & NA & NA & 13.8 \\
\hline Otherd & 4.5 & 12.9 & 5.2 & $1.7^{\mathrm{b}}$ \\
\hline \multicolumn{5}{|l|}{ Treatment length, weeks $^{e}$} \\
\hline 8 & $11.3^{\mathrm{a}}$ & $\leq 1.0^{\mathrm{a}}$ & 10.3 & $23.4^{\mathrm{c}}$ \\
\hline 12 & $72.7^{\mathrm{a}}$ & $71.0^{\mathrm{a}}$ & 72.6 & 69.0 \\
\hline 16 & $\leq 1.0^{\mathrm{a}}$ & $3.2^{\mathrm{a}}$ & 1.1 & $1.1^{\mathrm{c}}$ \\
\hline 24 & $15.1^{\mathrm{a}}$ & $25.8^{\mathrm{a}}$ & 16.0 & $6.5^{c}$ \\
\hline Concomitant ribavirin & 49.8 & 67.7 & 51.4 & $29.0^{\mathrm{c}}$ \\
\hline \multicolumn{5}{|l|}{ Clinical characteristics, \% } \\
\hline \multicolumn{5}{|l|}{ Genotype } \\
\hline 1 & 48.4 & 6.3 & 47.3 & $59.9^{c}$ \\
\hline 2 & 9.5 & 3.2 & 9.0 & $15.4^{\mathrm{c}}$ \\
\hline 3 & 9.5 & 16.1 & 10.1 & $11.0^{c}$ \\
\hline 4 & $\leq 1.0$ & $\leq 1.0$ & $\leq 1.0$ & $\leq 1.0^{c}$ \\
\hline Missing & 31.8 & 45.2 & 32.9 & $13.0^{c}$ \\
\hline \multicolumn{5}{|l|}{ Fibrosis score } \\
\hline 1 & $\leq 1.0^{\mathrm{a}}$ & $\leq 1.0^{\mathrm{a}}$ & $\leq 1.0$ & $\leq 1.0^{\mathrm{c}}$ \\
\hline 2 & $20.2^{\mathrm{a}}$ & $6.5^{\mathrm{a}}$ & 19.0 & $32.9^{c}$ \\
\hline 3 & $16.9^{a}$ & $3.2^{\mathrm{a}}$ & 15.8 & $19.1^{\mathrm{c}}$ \\
\hline 4 & $29.4^{a}$ & $45.2^{\mathrm{a}}$ & 30.7 & $33.3^{c}$ \\
\hline Missing & $32.9^{\mathrm{a}}$ & $45.2^{\mathrm{a}}$ & 34.0 & $13.0^{\mathrm{c}}$ \\
\hline \multicolumn{5}{|l|}{ Treatment experience } \\
\hline Treatment naive & 48.4 & 35.5 & 47.3 & $71.3^{\mathrm{c}}$ \\
\hline Treatment experienced & 8.6 & 6.5 & 8.4 & $11.2^{\mathrm{c}}$ \\
\hline Missing & 43.0 & 58.1 & 44.3 & $17.5^{\mathrm{c}}$ \\
\hline Median initial viral load (IQR, IU/mL) & $\begin{array}{c}1,774,185 \\
(403,305, \\
5,028,680)\end{array}$ & $\begin{array}{c}1,190,000 \\
(428,000 \\
2,573,059)\end{array}$ & $\begin{array}{c}1,705,855 \\
(409,714 \\
4,930,000) \\
\end{array}$ & $\begin{array}{l}1,392,870 \\
(461,080, \\
3,413,720)\end{array}$ \\
\hline Deyo-Charlson Comorbidity Index, mean (SD) & $3.1(2.8)^{\mathrm{b}}$ & $4.5(2.5)^{\mathrm{b}}$ & $3.2(2.8)$ & $2.8(2.5)$ \\
\hline
\end{tabular}




\section{Sustained Virologic Response and Costs Associated with Direct-Acting Antivirals for Chronic Hepatitis C Infection in Oklahoma Medicaid}

TABLE 1 Baseline and Clinical Characteristics of Oklahoma Medicaid Members Receiving DAA Treatment and Evaluated for SVR12 (continued)

\begin{tabular}{|c|c|c|c|c|}
\hline & $\begin{array}{l}\text { SVR12 } \\
\text { Success } \\
(\mathrm{n}=337)\end{array}$ & $\begin{array}{l}\text { SVR12 } \\
\text { Failure } \\
(\mathrm{n}=31)\end{array}$ & $\begin{array}{c}\text { Overall } \\
(\mathrm{n}=368)\end{array}$ & $\begin{array}{l}\text { Not Evaluated } \\
\text { for SVR12 } \\
(\mathrm{n}=538)\end{array}$ \\
\hline \multicolumn{5}{|l|}{ Comorbidities, $\%$} \\
\hline Myocardial infarction & 5.3 & 3.2 & 5.2 & 3.9 \\
\hline Congestive heart failure & 10.7 & 16.1 & 11.1 & 7.4 \\
\hline Peripheral vascular disease & 5.9 & 9.7 & 6.3 & 6.3 \\
\hline Cerebrovascular disease & 7.1 & 6.5 & 7.1 & 5.6 \\
\hline Dementia & $\leq 1.0$ & $\leq 1.0$ & $\leq 1.0$ & 1.3 \\
\hline Chronic pulmonary disease & 43.6 & 51.6 & 44.3 & 40.9 \\
\hline Rheumatic disease & 10.7 & $\leq 1.0$ & 9.8 & 8.0 \\
\hline Peptic ulcer disease & 6.2 & 12.9 & 6.8 & $3.4^{\mathrm{a}}$ \\
\hline Mild liver disease & $59.9^{b}$ & $83.9^{b}$ & 62.0 & $75.3^{c}$ \\
\hline Moderate or severe liver disease & $22.0^{c}$ & $58.1^{\mathrm{c}}$ & 25.0 & $16.0^{c}$ \\
\hline Diabetes without chronic complication & 26.1 & 38.7 & 27.2 & 23.8 \\
\hline Diabetes with chronic complication & 7.4 & 6.5 & 7.3 & 8.2 \\
\hline Hemiplegia or paraplegia & 2.1 & 3.2 & 2.2 & 1.3 \\
\hline Renal disease & 5.9 & 9.7 & 6.3 & 5.4 \\
\hline Any malignancy, excluding malignant neoplasm of skin & 7.1 & 6.5 & 7.1 & 5.8 \\
\hline Metastatic solid tumor & $\leq 1.0$ & $\leq 1.0$ & $\leq 1.0$ & $\leq 1.0$ \\
\hline HIV/AIDS & 2.1 & $\leq 1.0$ & 1.9 & 2.0 \\
\hline Mild liver diagnoses, $\%$ & $50.5^{\mathrm{b}}$ & $77.4^{\mathrm{b}}$ & 52.7 & $43.1^{\mathrm{b}}$ \\
\hline Alcoholic cirrhosis & $10.4^{\mathrm{a}}$ & $25.8^{\mathrm{a}}$ & 11.7 & $6.1^{\mathrm{b}}$ \\
\hline Nonalcoholic cirrhosis & $41.3^{c}$ & $77.4^{\mathrm{c}}$ & 44.3 & $33.1^{\mathrm{c}}$ \\
\hline Biliary cirrhosis & $\leq 1.0$ & 3.2 & $\leq 1.0$ & $\leq 1.0$ \\
\hline Chronic hepatitis, nonviral & 18.4 & 9.7 & 17.7 & 13.9 \\
\hline Moderate to severe liver diagnoses, $\%$ & $29.1^{\mathrm{c}}$ & $58.1^{\mathrm{c}}$ & 31.5 & $24.5^{\mathrm{a}}$ \\
\hline Hepatocellular carcinoma & 2.4 & $\leq 1.0$ & 2.2 & $\leq 1.0$ \\
\hline Esophageal varices & 14.0 & 22.6 & 14.7 & $8.6^{\mathrm{b}}$ \\
\hline Hepatic encephalopathy & 7.7 & 16.1 & 8.4 & 6.0 \\
\hline Portal hypertension & $15.7^{\mathrm{c}}$ & $48.4^{c}$ & 18.5 & $10.2^{c}$ \\
\hline Hepatorenal syndrome & 1.2 & $<1.0$ & 1.1 & $\leq 1.0$ \\
\hline Other sequelae of chronic liver disease & 5.0 & 9.7 & 5.4 & 5.0 \\
\hline Gastrointestinal bleed & 8.3 & 6.5 & 8.2 & 8.2 \\
\hline Ascites & $11.3^{b}$ & $32.3^{\mathrm{b}}$ & 13.0 & $8.7^{\mathrm{a}}$ \\
\hline Liver transplant & 2.7 & $\leq 1.0$ & 2.5 & $\leq 1.0$ \\
\hline Cirrhosis, $\%$ & $41.3^{c}$ & $77.4^{\mathrm{c}}$ & 44.3 & $34.0^{\mathrm{b}}$ \\
\hline Decompensated cirrhosis ${ }^{f}$ & $24.3^{c}$ & $58.1^{\mathrm{c}}$ & 27.2 & $18.0^{\mathrm{b}}$ \\
\hline Compensated cirrhosis (mutually exclusive from decompensated)g & 16.9 & 19.4 & 17.1 & 16.0 \\
\hline \multicolumn{5}{|l|}{ Adherence } \\
\hline MPR, mean (SD) & $0.98(0.10)$ & $0.99(0.03)$ & $0.98(0.10)$ & $0.91(0.21)^{c}$ \\
\hline $\mathrm{MPR} \geq 0.80, \%$ & 95.0 & 100.0 & 95.4 & $81.2^{\mathrm{c}}$ \\
\hline Proportion with > 3-day gap between monthly prescriptions, $\%$ & 46.6 & 41.9 & 46.2 & $31.6^{\mathrm{c}}$ \\
\hline Sum of gap days, mean (SD) & $5.2(9.6)$ & $6.9(12.1)$ & $5.3(9.8)$ & $5.1(13.6)^{\mathrm{a}}$ \\
\hline Proportion who discontinued treatment early, \% & 5.9 & 6.5 & 6.0 & $18.8^{\mathrm{c}}$ \\
\hline At least 1 hospitalization or ED visit in 12 months before treatment initiation, $\%$ & 54.0 & 64.5 & 54.9 & $62.3^{\mathrm{a}}$ \\
\hline At least 1 hospitalization or ED visit in 12 months after treatment initiation, \% & 55.5 & 64.5 & 56.3 & $41.5^{\mathrm{c}}$ \\
\hline Restarted therapy after failure and achieved SVR, \% & NA & 22.6 & 1.9 & NA \\
\hline Restarted therapy after failure and had unknown SVR, \% & NA & 25.8 & 2.2 & NA \\
\hline
\end{tabular}

$a P<0.050$

${ }^{b} P<0.010$

${ }^{c} P<0.001$

Note: P values were calculated from Wilcoxon-Mann-Whitney tests for nonparametric independent continuous variables; chi-square goodness of fit tests for categorical

variables; and Fisher's exact tests for categorical variables where expected cell counts were $\leq 5$.

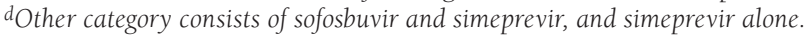

eTreatment lengths (12 or 24 weeks) were imputed for preamended prior authorization members based on prescription claims.

fAlcoholic cirrhosis, nonalcoholic cirrhosis, or biliary cirrhosis and hepatocellular carcinoma, hepatic encephalopathy, esophageal varices with or without bleeding, portal hypertension, hepatorenal syndrome, gastrointestinal bleeding, ascites, liver transplant, and other sequelae of advanced liver disease.

gAlcoholic cirrhosis, nonalcoholic cirrhosis, or biliary cirrhosis in patients without decompensated cirrhosis.

$D A A=$ direct-acting antiviral; $E D=$ emergency department; HIV/AIDS = human immunodeficiency virus/acquired immune deficiency syndrome; IQR =interquartile range; $M P R=$ medication possession ratio; $N A=$ not applicable; $S D=$ standard deviation; $S V R 12=$ sustained virological response at 12 weeks. 


\section{Sustained Virologic Response and Costs Associated with Direct-Acting Antivirals for Chronic Hepatitis C Infection in Oklahoma Medicaid}

TABLE 2 Pre- and Post-Index Utilization and Costs for Oklahoma Medicaid Members Receiving DAA Treatment and Reported SVR12

\begin{tabular}{|c|c|c|c|c|c|c|c|c|c|}
\hline & \multicolumn{3}{|c|}{ SVR12 Success $(\mathrm{n}=337)$} & \multicolumn{3}{|c|}{ SVR12 Failure $(n=31)$} & \multicolumn{3}{|c|}{ Overall $(n=368)$} \\
\hline & Pre & Post & $P$ Value $^{\mathrm{a}}$ & Pre & Post & $P$ Value $^{\mathrm{a}}$ & Pre & Post & $P$ Value ${ }^{a}$ \\
\hline \multicolumn{10}{|l|}{ Utilization } \\
\hline $\begin{array}{l}\text { Inpatient hospitalizations PM, } \\
\text { mean (SD) }\end{array}$ & $\begin{array}{c}0.4 \\
(1.1)\end{array}$ & $\begin{array}{c}0.4 \\
(1.2)\end{array}$ & 0.324 & $\begin{array}{c}0.4 \\
(0.7) \\
\end{array}$ & $\begin{array}{c}0.7 \\
(1.5)\end{array}$ & 0.432 & $\begin{array}{c}0.4 \\
(1.1)\end{array}$ & $\begin{array}{l}0.4 \\
(1.2)\end{array}$ & 0.557 \\
\hline $0, \mathrm{n}(\%)$ & 78.6 & 78.3 & 1.000 & 71.0 & 67.7 & 1.000 & 78.0 & 77.5 & 0.917 \\
\hline $1, \mathrm{n}(\%)$ & 13.4 & 15.7 & & 19.4 & 19.4 & & 13.9 & 16.0 & \\
\hline$\geq 2, \mathrm{n}(\%)$ & 8.0 & 5.9 & & 9.7 & 12.9 & & 8.2 & 6.5 & \\
\hline ED visits PM, mean (SD) & $\begin{array}{c}1.6 \\
(2.6) \\
\end{array}$ & $\begin{array}{l}1.5 \\
(2.8)\end{array}$ & 0.266 & $\begin{array}{c}1.7 \\
(2.3) \\
\end{array}$ & $\begin{array}{l}2.2 \\
(2.1)\end{array}$ & 0.082 & $\begin{array}{c}1.6 \\
(2.5) \\
\end{array}$ & $\begin{array}{l}1.6 \\
(2.7)\end{array}$ & 0.579 \\
\hline $0, \mathrm{n}(\%)$ & 49.0 & 48.7 & 1.000 & 41.9 & 35.5 & 0.754 & 48.4 & 47.6 & 0.862 \\
\hline $1, \mathrm{n}(\%)$ & 16.3 & 21.1 & & 25.8 & 6.5 & & 17.1 & 19.8 & \\
\hline$\geq 2, \mathrm{n}(\%)$ & 34.7 & 30.3 & & 32.3 & 58.1 & & 34.5 & 32.6 & \\
\hline $\begin{array}{l}\text { Physician office visits PM, } \\
\text { mean (SD) }\end{array}$ & $\begin{array}{l}12.2 \\
(8.8)\end{array}$ & $\begin{array}{l}12.6 \\
(8.9)\end{array}$ & 0.557 & $\begin{array}{l}10.9 \\
(5.6)\end{array}$ & $\begin{array}{l}13.1 \\
(6.6)\end{array}$ & 0.010 & $\begin{array}{l}12.2 \\
(8.6)\end{array}$ & $\begin{array}{l}12.6 \\
(8.7)\end{array}$ & 0.229 \\
\hline $0, \mathrm{n}(\%)$ & 2.4 & 2.7 & 1.000 & $<1.0$ & $\leq 1.0$ & NA & 2.2 & 2.5 & 0.633 \\
\hline $1, \mathrm{n}(\%)$ & $\leq 1.0$ & $\leq 1.0$ & & 3.2 & $\leq 1.0$ & & $\leq 1.0$ & $\leq 1.0$ & \\
\hline$\geq 2, \mathrm{n}(\%)$ & 97.3 & 96.4 & & 96.8 & 100.0 & & 97.3 & 96.7 & \\
\hline \multicolumn{10}{|l|}{ Costs, $\$$ b } \\
\hline Combined total expenditure & $6,588,852$ & $46,022,698$ & $<0.001$ & 724,242 & $5,601,587$ & $<0.001$ & $7,313,094$ & $51,624,285$ & $<0.001$ \\
\hline $\begin{array}{l}\text { Combined expenditure PM, } \\
\text { mean (SD) }\end{array}$ & $\begin{array}{c}19,551 \\
(28,823) \\
\end{array}$ & $\begin{array}{r}136,566 \\
(50,271)\end{array}$ & $<0.001$ & $\begin{array}{l}23,363 \\
(24,033)\end{array}$ & $\begin{array}{l}180,696 \\
(62,690) \\
\end{array}$ & $<0.001$ & $\begin{array}{c}19,872 \\
(28,442)\end{array}$ & $\begin{array}{c}140,283 \\
(52,779) \\
\end{array}$ & $<0.001$ \\
\hline $\begin{array}{l}\text { Combined expenditure PMPM, } \\
\text { mean (SD) }\end{array}$ & $\begin{array}{l}1,629 \\
(2,402)\end{array}$ & $\begin{array}{l}11,380 \\
(4,189)\end{array}$ & $<0.001$ & $\begin{array}{l}1,947 \\
(2,003)\end{array}$ & $\begin{array}{l}15,058 \\
(5,224)\end{array}$ & $<0.001$ & $\begin{array}{l}1,514 \\
(2,157)\end{array}$ & $\begin{array}{l}11,690 \\
(4,398)\end{array}$ & $<0.001$ \\
\hline Total medical expenditure & $4,738,861$ & $3,930,741$ & 0.056 & 418,666 & 605,738 & 0.291 & $5,157,527$ & $4,536,479$ & 0.174 \\
\hline $\begin{array}{l}\text { Medical expenditure PM, } \\
\text { mean (SD) }\end{array}$ & $\begin{array}{l}14,062 \\
(24,174) \\
\end{array}$ & $\begin{array}{l}11,664 \\
(17,990)\end{array}$ & $<0.001$ & $\begin{array}{c}13,505 \\
(13,900)\end{array}$ & $\begin{array}{c}19,540 \\
(30,956)\end{array}$ & 0.760 & $\begin{array}{c}14,015 \\
(23,470)\end{array}$ & $\begin{array}{l}12,327 \\
(19,479)\end{array}$ & $<0.001$ \\
\hline $\begin{array}{l}\text { Medical expenditure PMPM, } \\
\text { mean (SD) }\end{array}$ & $\begin{array}{c}1,071 \\
(2,014)\end{array}$ & $\begin{array}{l}971.99 \\
(1,499) \\
\end{array}$ & $<0.001$ & $\begin{array}{c}1,125 \\
(1,158) \\
\end{array}$ & $\begin{array}{c}1,628 \\
(2,580) \\
\end{array}$ & 0.760 & $\begin{array}{c}1,168 \\
(1,956) \\
\end{array}$ & $\begin{array}{c}1,027 \\
(1,623) \\
\end{array}$ & $<0.001$ \\
\hline Total pharmacyc expenditure & $1,849,991$ & $42,091,957$ & $<0.001$ & 305,576 & $4,995,849$ & $<0.001$ & $1,974,977$ & $42,946,887$ & $<0.001$ \\
\hline $\begin{array}{l}\text { Pharmacyc expenditure PM, } \\
\text { mean (SD) }\end{array}$ & $\begin{array}{c}5,489 \\
(14,111) \\
\end{array}$ & $\begin{array}{c}124,902 \\
(45,381)\end{array}$ & $<0.001$ & $\begin{array}{c}9,857 \\
(19,996) \\
\end{array}$ & $\begin{array}{l}161,156 \\
(65,870)\end{array}$ & $<0.001$ & $\begin{array}{c}5,367 \\
(13,486) \\
\end{array}$ & $\begin{array}{c}116,704 \\
(43,461)\end{array}$ & $<0.001$ \\
\hline $\begin{array}{l}\text { Pharmacyc expenditure PMPM, } \\
\text { mean (SD) }\end{array}$ & $\begin{array}{c}457 \\
(1,176) \\
\end{array}$ & $\begin{array}{l}10,408 \\
(3,782)\end{array}$ & $<0.001$ & $\begin{array}{c}821 \\
(1,667) \\
\end{array}$ & $\begin{array}{l}13,430 \\
(5,489)\end{array}$ & $<0.001$ & $\begin{array}{c}447 \\
(1,124) \\
\end{array}$ & $\begin{array}{c}9,725 \\
(3,622)\end{array}$ & $<0.001$ \\
\hline $\begin{array}{l}\text { Total HCVd pharmacy } \\
\text { expenditure }\end{array}$ & 310,251 & $40,218,357$ & $<0.001$ & 115,180 & $4,781,827$ & $<0.001$ & 425,432 & $45,000,185$ & $<0.001$ \\
\hline $\begin{array}{l}\text { HCVd pharmacy expenditure } \\
\text { PM, mean (SD) }\end{array}$ & $\begin{array}{c}921 \\
(7,298)\end{array}$ & $\begin{array}{l}119,342 \\
(43,831)\end{array}$ & $<0.001$ & $\begin{array}{c}3,715 \\
(14,339) \\
\end{array}$ & $\begin{array}{l}154,253 \\
(64,387) \\
\end{array}$ & $<0.001$ & $\begin{array}{c}1,156 \\
(8,134)\end{array}$ & $\begin{array}{l}122,283 \\
(46,819)\end{array}$ & $<0.001$ \\
\hline $\begin{array}{l}\text { HCVd pharmacy expenditure } \\
\text { PMPM, mean (SD) }\end{array}$ & $\begin{array}{c}77 \\
(608) \\
\end{array}$ & $\begin{array}{c}9,945 \\
(3,653) \\
\end{array}$ & $<0.001$ & $\begin{array}{c}310 \\
(1,195) \\
\end{array}$ & $\begin{array}{l}12,854 \\
(5,366) \\
\end{array}$ & $<0.001$ & $\begin{array}{c}96 \\
(678) \\
\end{array}$ & $\begin{array}{l}10,190 \\
(3,902)\end{array}$ & $<0.001$ \\
\hline $\begin{array}{l}\text { Total non-HCV pharmacy } \\
\text { expenditure }\end{array}$ & $1,539,740$ & $1,873,599$ & 0.012 & 190,396 & 214,022 & 0.506 & $1,730,135$ & $2,087,621$ & 0.009 \\
\hline $\begin{array}{l}\text { Non-HCV pharmacy expenditure } \\
\text { PM, mean (SD) }\end{array}$ & $\begin{array}{c}4,569 \\
(11,683) \\
\end{array}$ & $\begin{array}{c}5,560 \\
(12,238) \\
\end{array}$ & $<0.001$ & $\begin{array}{c}6,142 \\
(7,574)\end{array}$ & $\begin{array}{l}6,904 \\
(7,188)\end{array}$ & 0.397 & $\begin{array}{c}4,701 \\
(11,395) \\
\end{array}$ & $\begin{array}{c}5,673 \\
(11,894) \\
\end{array}$ & $<0.001$ \\
\hline $\begin{array}{l}\text { Non-HCV pharmacy expenditure } \\
\text { PMPM, mean (SD) }\end{array}$ & $\begin{array}{l}381 \\
(974)\end{array}$ & $\begin{array}{c}463 \\
(1,020)\end{array}$ & $<0.001$ & $\begin{array}{l}512 \\
(631)\end{array}$ & $\begin{array}{c}575 \\
(599)\end{array}$ & 0.397 & $\begin{array}{c}392 \\
(950)\end{array}$ & $\begin{array}{l}473 \\
(991)\end{array}$ & $<0.001$ \\
\hline
\end{tabular}

a $P$ values were calculated from Wald tests for totals, Wilcoxon signed rank sum tests for difference in means for dependent nonparametric continuous variables, and McNemar tests for dependent categorical variables.

bxpenditures in the posttreatment initiation period included the paid claim on the index date.

cExpenditures were for all medication claims, including nonhepatitis $C$ treatment.

${ }^{d}$ Expenditures were for sofosbuvir, simeprevir, ledipasvir/sofosbuvir, ombitasvir/paritaprevir/ritonavir and dasabuvir, elbasvir/grazoprevir, telaprevir, boceprevir, pegylated interferon, and ribavirin.

$D A A=$ direct-acting antiviral; $E D=$ emergency department; $H C V=$ hepatitis $C$ virus; $N A=$ not applicable; $P M=$ per member; $P M P M=$ per member per month; $S D=$ standard deviation; SVR12= sustained virological response at 12 weeks. 


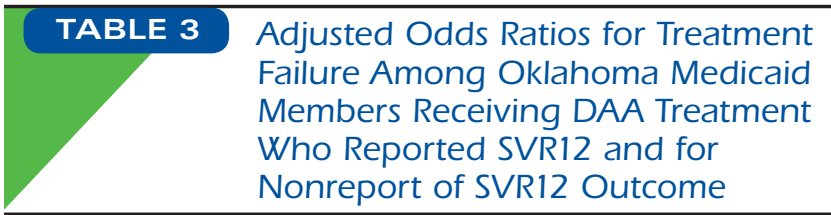

\begin{tabular}{|c|c|c|}
\hline & $\begin{array}{c}\text { Adjusted Odds } \\
\text { of SVR12 Failure } \\
\text { Among All Members } \\
\text { with Reported } \\
\text { SVR12 Result } \\
(\mathrm{n}=368)\end{array}$ & $\begin{array}{l}\text { Adjusted Odds of } \\
\text { Nonreporting of } \\
\text { SVR12 Among All } \\
\text { Members Receiving } \\
\text { DAA Therapy } \\
(\mathrm{n}=906)\end{array}$ \\
\hline & OR $(95 \% \mathrm{CI})$ & OR $(95 \% \mathrm{CI})$ \\
\hline \multicolumn{3}{|l|}{ Member characteristics } \\
\hline Age & $0.98(0.93-1.03)$ & $0.98 \quad(0.97-1.00)$ \\
\hline Male sex & $3.34^{\mathrm{a}}(1.31-8.55)$ & $0.90(0.65-1.23)$ \\
\hline Race (vs. white) & $1.12(0.42-3.01)$ & $0.93 \quad(0.62-1.39)$ \\
\hline $\begin{array}{l}\text { Rural/metropolitan residence } \\
\text { (vs. metropolitan) }\end{array}$ & $0.80(0.34-1.83)$ & $1.37(0.99-1.88)$ \\
\hline \multicolumn{3}{|c|}{ Treatment regimens $($ sofosbuvir $=$ referent $)$} \\
\hline Ledipasvir/sofosbuvir & $0.59(0.19-1.84)$ & $3.53^{\mathrm{a}}(2.49-5.02)$ \\
\hline $\begin{array}{l}\text { Other (DCV, SOF/SMV, SMV, } \\
\text { or 3D regimen) }\end{array}$ & $0.68(0.19-2.39)$ & $7.72^{\mathrm{b}}(4.77-12.50)$ \\
\hline \multicolumn{3}{|l|}{ Comorbidities } \\
\hline $\begin{array}{l}\text { Modified Deyo-Charlson } \\
\text { Comorbidity Index }\end{array}$ & $0.98(0.80-1.19)$ & $0.93(0.85-1.01)$ \\
\hline $\begin{array}{l}\text { Decompensated cirrhosis } \\
\text { (vs. no decompensated } \\
\text { cirrhosis) }\end{array}$ & $6.69^{\mathrm{b}}(2.62-17.10)$ & $0.48^{\mathrm{b}}(0.32-0.72)$ \\
\hline $\begin{array}{l}\text { Compensated cirrhosis } \\
\text { (mutually exclusive from } \\
\text { decompensated vs. no } \\
\text { compensated cirrhosis)e }^{\text {e }}\end{array}$ & $3.52^{\mathrm{c}}(1.11-11.14)$ & $0.70^{c}(0.39-0.89)$ \\
\hline $\begin{array}{l}\text { At least } 1 \text { hospitalization or } \\
\text { ED visit before treatment }\end{array}$ & $1.67(0.68-4.11)$ & $1.26(0.92-1.74)$ \\
\hline $\begin{array}{l}\text { Initiated treatment after } \\
\text { amendments to prior } \\
\text { authorization program }\end{array}$ & $0.48 \quad(0.16-1.43)$ & - \\
\hline $\mathrm{MPR} \geq 0.95$ & $1.83(0.23-14.85)$ & $0.18^{\mathrm{b}}(0.10-0.32)$ \\
\hline
\end{tabular}

Note: Odds ratios were estimated from logistic regression models with Huber-White robust standard errors. AUROC: 0.79; Log likelihood: -91.17; Prob>chi-square: $<0.001$. ${ }^{a} P<0.010$.

${ }^{b} P<0.001$

${ }^{c} P<0.050$.

${ }^{d}$ Alcoholic cirrhosis, nonalcoholic cirrhosis, or biliary cirrhosis and hepatocellular carcinoma, hepatic encephalopathy, esophageal varices with or without bleeding, portal hypertension, hepatorenal syndrome, gastrointestinal bleeding, ascites, liver transplant, or other sequelae of advanced liver disease.

eAlcoholic cirrhosis, nonalcoholic cirrhosis, or biliary cirrhosis in patients without decompensated cirrhosis.

$3 D=$ paritaprevir/ritonavir/ombitasvir/dasabuvir; $A U R O C=$ area under receiver operating curve; $D A A=$ direct-acting antiviral; $D C V=$ daclatasvir; $E D=$ emergency department; $M P R=$ medication possession ratio; $O R=$ odds ratio; $S M V=$ simeprevir; $\mathrm{SOF}=$ sofosbuvir; SVR12 = sustained virological response at 12 weeks.

\section{Multivariable Analysis of Treatment Failure and Cost}

Table 3 presents the results of the multivariable analyses of SVR12 failure that controlled for demographics, clinical attributes, comorbidities, HCV regimens, adherence, and health resource utilization. The presence of cirrhosis was associated with markedly higher odds of treatment failure, independent of other factors: The adjusted odds of treatment failure for members with decompensated cirrhosis was 6.69 times higher (95\% $\mathrm{CI}=2.62-17.10)$ than for those with no decompensated cirrhosis. The adjusted odds of treatment failure for those with compensated cirrhosis was 3.52 times higher (95\% CI $=1.11-11$.14) than for those with no cirrhosis. Independent of the presence of cirrhosis, the adjusted odds of treatment failure was 3.34 times higher (95\% CI $=1.31-8.55$ ) among men than among women. No statistically significant differences were seen among treatment regimens when compared with sofosbuvir.

Analysis for the odds of SVR12 nonreport is also presented in Table 3. Controlling for previously mentioned variables, those with decompensated cirrhosis had $-52 \%$ lower odds (95\% CI $=0.32-0.72$ ) of SVR12 nonreport compared with those without decompensated cirrhosis, and those with compensated cirrhosis had $-30 \%$ lower odds (95\% CI=0.39-0.89) of SVR12 nonreport compared with those without compensated cirrhosis.

Concerning costs, Table 4 presents the results of a multivariable analysis of ratios of combined total expenditures following DAA treatment initiation. Across the overall cohort, the ledipasvir/sofosbuvir combination was associated with significantly lower expenditures of $-19 \%$ (exponentiated beta, $\exp [b]=0.81$, 95\% CI $=0.74-0.89)$ compared with sofosbuvir, while other regimens incurred $+18 \%$ higher expenditures $(\exp [b]=1.18$, 95\% CI=1.03-1.35) than those who received sofosbuvir. Decompensated cirrhosis was associated with significantly increased expenditures of $+20 \%(\exp [b]=1.20,95 \% \mathrm{CI}=$ 1.09-1.31) compared with the group with no cirrhosis. Costs were also $+18 \%$ higher among those for whom treatment failed $(\exp [b]=1.18,95 \% \mathrm{CI}=1.04-1.34)$ than for those who experienced treatment success.

Sensitivity analyses that varied the definitions of cirrhosis and MPR thresholds showed no differences in results.

\section{Discussion}

This investigation of Medicaid beneficiaries with HCV assessed DAA treatment success, SVR12 reporting, and direct medical cost within a real-world setting. Although a majority of individuals who received DAAs did not have a reported SVR (538/906, $59.4 \%$ ), a high percentage of those with reported viral loads achieved treatment success $(337 / 368,91.6 \%)$. Independent of other factors, a 6.69-times higher odds of treatment failure was associated with decompensated cirrhosis and a 3.52-times higher odds was observed for compensated types $(P<0.050)$. No difference was observed between either DAA treatments or medication adherence $<95 \%$, and males were associated with a higher odds of treatment failure $(P<0.050)$. Adjusted costs were lower among ledipasvir/sofosbuvir regimens, and both treatment failure and decompensated cirrhosis cases incurred higher adjusted costs $(P<0.050)$. 


\begin{tabular}{|c|c|}
\hline $\begin{array}{l}\text { Ratios of Total Post-In } \\
\text { Expenditures Among } \\
\text { Medicaid Members W } \\
\text { Treatment and Repor }\end{array}$ & $\begin{array}{l}\text { tion } \\
\text { klahoma } \\
\text { Received DAA } \\
\text { SVR12 }\end{array}$ \\
\hline & Overall \\
\hline & $\exp (b)(95 \% \mathrm{CI})$ \\
\hline Member characteristics & \\
\hline Age & $1.00 \quad(0.99-1.00)$ \\
\hline Male sex & $1.00 \quad(0.92-1.09)$ \\
\hline Race $($ white $=$ referent $)$ & \\
\hline Black & $0.90 \quad(0.80-1.09)$ \\
\hline Other & $1.05 \quad(0.90-1.24)$ \\
\hline Rural/metropolitan residence (vs. metropolitan) & $0.96 \quad(0.89-1.05)$ \\
\hline Regimen (sofosbuvir $=$ referent) & \\
\hline Ledipasvir/sofosbuvir & $0.81 \quad(0.74-0.89)^{\mathrm{b}}$ \\
\hline Other (DCV, SOF/SMV, SMV, or 3D regimen) & $1.18(1.03-1.35)^{\mathrm{c}}$ \\
\hline Comorbidities & \\
\hline Deyo-Charlson Comorbidity Index & $1.02(1.01-1.04)^{\mathrm{c}}$ \\
\hline $\begin{array}{l}\text { Decompensated cirrhosisd (vs. no } \\
\text { decompensated cirrhosis) }\end{array}$ & $1.20(1.09-1.31)^{\mathrm{b}}$ \\
\hline $\begin{array}{l}\text { Compensated cirrhosis (mutually exclusive from } \\
\text { decompensated vs. no compensated cirrhosis)e }\end{array}$ & $1.04 \quad(0.96-1.14)$ \\
\hline $\begin{array}{l}\text { Initiated treatment after amendments to the } \\
\text { prior authorization program }\end{array}$ & $1.00 \quad(0.90-1.12)$ \\
\hline $\mathrm{MPR} \geq 0.95$ & $1.23 \quad(0.95-1.60)$ \\
\hline Baseline costs (per $\$ 1,000)$ & $1.00 \quad(1.00-1.00)$ \\
\hline Treatment failure & $1.18 \quad(1.04-1.34)^{\mathrm{c}}$ \\
\hline 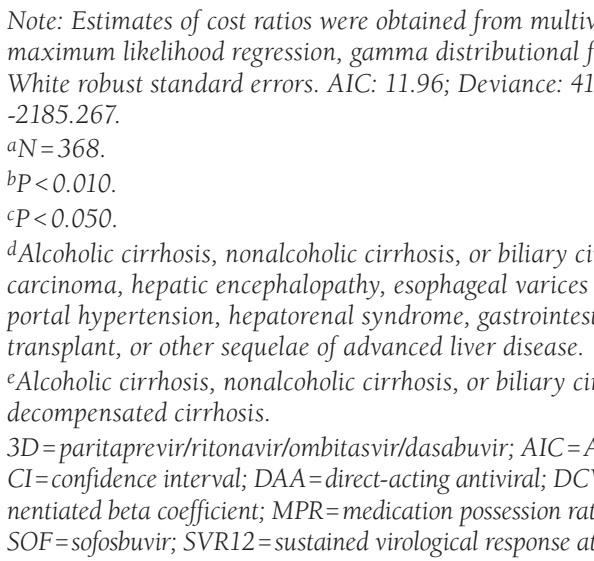 & $\begin{array}{l}\text { ible generalized } \\
\text { ly, log link, Huber- } \\
\text { Log pseudolikelihood: } \\
\text { sis and hepatocellular } \\
\text { or without bleeding, } \\
\text { bleeding, ascites, liver } \\
\text { sis in patients without } \\
\text { Information Criterion; } \\
\text { aclatasvir; exp }(b)=\operatorname{expo-} \\
\text { weeks. }\end{array}$ \\
\hline
\end{tabular}

The relatively large percentage of members (59.4\%) without any reported SVR12 is an important observation of this study. These individuals may have been lost to follow-up, discontinued treatment early, failed to achieve SVR12, or involved a combination of the above. Therefore, it may be anticipated that the current study is likely to have underestimated the SVR12 failure rate. Analysis of those without SVR12 reports showed that those with advanced liver sequelae (i.e., cirrhosis) were being retained in the HCV PA program, while those with milder or no liver sequelae were dropping out more often.
Additionally, even though the adherence rate was high among those with report

ed SVR12s $(0.98 \pm 0.10)$, the MPR among those without reported SVR12 was significantly lower at $0.91 \pm 0.21$ $(P<0.001)$. Although not systematically measured, anecdotal observations within the HCV PA program appeared to attribute nonreported SVR12s due to loss to patient follow-up at the clinic setting (e.g., Medicaid members did not return to their physicians for final viral load testing). As such, findings from the current study suggest that future research should elicit reasons from patients for this loss to follow-up or early discontinuation, and that interventions may be especially important to retain members with less severe liver sequelae. An interdisciplinary approach may be suggested for all patients to improve adherence, to minimize barriers for successful treatment, and to track final viral loads to assess clinical outcomes. ${ }^{29}$

The relatively high treatment success found in the current work (i.e., 91.6\% achieving SVR12) parallels the overall percentages of $86 \%-100 \%$ reported in the ION, COSMOS, OPTIMIST, and TURQUOISE clinical trials. ${ }^{30-34}$ Several other studies have examined SVR in real-world settings, with overall SVRs ranging from $82.4 \%$ to $92.8 \% .{ }^{35-39}$ Among $17,487 \mathrm{HCV}$ patients in the U.S. Veterans Affairs (VA) system treated with DAA regimens, Ioannou et al. (2016) observed a 92.8\% SVR12 (95\% CI $=92.3 \%-93.2 \%$ ) with a minimum and maximum of 65.7\%-90.6\% among patients with cirrhosis depending on viral genotype.$^{37}$ Backus et al. (2016) also examined DAA use (i.e., ledipasvir/sofosbuvir and ombitasvir/paritaprevir/ritonavir+dasabuvir) among 21,142 genotype 1 VA patients and reported SVR12 ranges of $87.8 \%-91.7 \%$ depending on treatment regimen. ${ }^{38}$ These authors also noted that decompensated liver disease was a negative predictor of SVR12 based on multivariable analyses.

Reddy et al. (2017) examined DAA treatments in 220 patients with advanced liver disease (Model for End-Stage Liver Disease [MELD] score of $\geq 10$ ) from medical centers in the United States, Germany, Israel, and Canada, reporting SVR12 from $35 \%$ to $84 \%$ depending on genotype, MELD score, and treatment regimen. ${ }^{40}$ While Reddy et al. reported lower SVR12s in cirrhotic cases than in the current study, these authors reported findings based on sofosbuvir or simeprevir therapies only. ${ }^{38}$ Shin et al. (2017) reported SVR12s of $75.0 \%$ 92.2\% among 343 patients with genotype 1 based in a Seattle, Washington, medical center, also noting that SVR12s among patients with cirrhosis were lower, and that female sex was a positive predictor of SVR12. ${ }^{33}$ Dalgard et al. (2017) found an overall SVR12 of 91\% among patients in Scandinavia and reported that cirrhosis and male sex were negative predictors of treatment success. ${ }^{39}$ The present study reinforces these findings, while also highlighting HCV treatment among Medicaid members, a notably vulnerable population. 


\section{Sustained Virologic Response and Costs Associated with Direct-Acting Antivirals for Chronic Hepatitis C Infection in Oklahoma Medicaid}

While the relationship between failure to achieve SVR12 and cirrhosis has been long observed, limited data exist concerning decompensated cirrhosis and DAA treatmentrelated outcomes. ${ }^{41-43}$ Historically, HCV patients with cirrhosis incurred lower SVR12 rates, higher risks of serious adverse events, and greater risks of sepsis, liver failure, and death. ${ }^{42}$ In an analysis of trial data for Child Class $C$ cirrhosis patients treated with DAAs, Guarino et al. (2017) reported that SVR12s were 50.0\%-96.0\%, with an average SVR12 across 10 studies of $74.9 \%$ (95\% CI $=65.6 \%-82.4 \%){ }^{43}$ In the current study, while SVR12 among cirrhotic patients was relatively high at $85.3 \%$, a need to ascertain the severity of liver disease appears warranted, as more assertive overall clinical management may be required in these cases. From a broader policy or public health stance, expansion of access to treatment should be considered for HCV patients without advanced liver sequelae, in order to prevent progression and possible deleterious effects on SVR12 achievement.

A significantly higher odds of treatment failure was observed among males $(\mathrm{OR}=3.34)$, independent of other factors in the current work $(P<0.050)$. Similar findings have been observed in other studies, including Dalgard et al. among Scandinavian countries for genotype 3, Shin et al. in genotype 1 patients based in Seattle, and the ALLY-3 study conducted by Nelson et al. (2015). ${ }^{35,39,44}$ Limited work has focused on the possible factors surrounding this association, although biological differences have been implicated as playing a role (i.e., a higher rate of spontaneous clearance of HCV may occur in women) as have behavioral factors (e.g., men are more likely to smoke and drink alcohol more frequently, which may negatively affect the course of disease). ${ }^{43-46}$

\section{Limitations}

Despite findings of this investigation of DAAs using both administrative claims and clinical data among members of Medicaid with HCV, certain limitations should be considered. Administrative claims data do not include information on MELD scores or Child Turcotte Pugh classifications. Proxy definitions of cirrhosis were determined through ICD-9-CM and ICD-10-CM codes, which may have underestimated the true prevalence of severe liver disease. No data on indirect costs or costs borne by the member were available or estimated; expenditures in claims data reflect amounts reimbursed by Medicaid. Treatment-related rebates were also not included, and final costs to the payer may be substantially lower than those reported. While researcher imputation of treatment length for pre-PA members may have overstated MPRs, only 12 weeks and 24 weeks were recommended at the time before PA implementation. Therefore, any variation based on days supply should have been minimized. Although algorithms were implemented to mitigate the effects of outliers due to the relatively low number of observations for SVR failures (i.e., Huber-White robust standard errors), statistical inference may still remain susceptible.

\section{Conclusions}

The present study provides real-world evidence of the clinical and economic impact of the second-generation DAAs in a state Medicaid program. High loss to follow-up was observed. Among those not lost to follow-up, high SVR12 achievement rates were seen even among those with severe liver disease, although cirrhotic disease was associated with large and significant odds of SVR12 treatment failure and increased costs. Continued work should be directed at addressing loss to follow-up and minimizing those HCV cases that manifest with advanced sequelae, potentially including a removal of barriers that impede either continued follow-up or treatment completion among already vulnerable populations.

\section{Authors}

TIMOTHY T. PHAM, PharmD, PhD; SHELLIE L. KEAST, PharmD, PhD; KEVIN C. FARMER, RPh, PhD; R. CHRIS RATHBUN, PharmD, BCPS, AQ-ID; BETHANY P. HOLDERREAD, PharmD; and GRANT H. SKREPNEK, PhD, RPh, University of Oklahoma College of Pharmacy, Oklahoma City. DAVID M. THOMPSON, PhD, PT, University of Oklahoma College of Public Health, Oklahoma City, and NANCY J. NESSER, PharmD, JD, Oklahoma Health Care Authority, Oklahoma City.

AUTHOR CORRESPONDENCE: Timothy T. Pham, PharmD, PhD, University of Oklahoma College of Pharmacy, ORI W-4403,

P.O. Box 26901, Oklahoma City, OK 73126-0901.

Tel.: 405.271.9039, ext. 31492; E-mail: timothy-pham@ouhsc.edu.

\section{DISCLOSURES}

No outside funding supported this study. Pham, Keast, Holderread, Nesser, and Skrepnek disclose either employment by the Oklahoma Health Care Authority or contractual work for this employer. Pham discloses fellowship funding from Purdue Pharma unrelated to this study. Keast and Skrepnek disclose research grant funding from Gilead Sciences and Abbvie. Holderread also reports grant funding from Gilead Sciences and fees from PRIME Education. Thompson, Farmer, and Rathbun have nothing to disclose.

\section{REFERENCES}

1. Ly KN, Xing J, Klevens RM, Jiles RB, Ward JW, Holmberg SD. The increasing burden of mortality from viral hepatitis in the United States between 1999 and 2007. Ann Intern Med. 2012;156(4):271-78.

2. Denniston MM, Jiles RB, Drobeniuc J, et al. Chronic hepatitis $C$ virus infection in the United States, National Health and Nutrition Examination Survey 2003 to 2010. Ann Intern Med. 2014;160(5):293-300.

3. Centers for Disease Control and Prevention. Hepatitis C FAQs for health professionals. July 17, 2014. Available at: http://www.cdc.gov/hepatitis/HCV/ HCVfaq.htm\#bl. Accessed April 25, 2018. 


\section{Sustained Virologic Response and Costs Associated with Direct-Acting Antivirals for Chronic Hepatitis C Infection in Oklahoma Medicaid}

4. Chen SL, Morgan TR. The natural history of hepatitis C virus (HCV) infection. Int J Med Sci. 2006;3(2):47-52.

5. El Khoury AC, Klimack WK, Wallace C, Razavi H. Economic burden of hepatitis C-associated diseases in the United States. J Viral Hepat. 2012;19(3):153-60

6. Xu F, Tong X, Leidner AJ. Hospitalizations and costs associated with hepatitis $C$ and advanced liver disease continue to increase. Health Aff (Millwood). 2014;33(10):1728-35.

7. Galbraith JW, Donnelly JP, Franco RA, Overton ET, Rodgers JB, Wang HE. National estimates of healthcare utilization by individuals with hepatitis $C$ virus infection in the United States. Clin Infect Dis. 2014;59(6):755-64.

8. Welsch C, Zeuzem S. Treatment of hepatitis C. In: Thomas HC, Lok ASF, Locarnini SA, Zuckerman AJ, eds. Viral Hepatitis. 4th ed. Oxford, UK: John Wiley; 2014:362-76.

9. Chou R, Carson S, Chan BKS, Care B. Drug class review: pegylated interferons for chronic hepatitis $\mathrm{C}$ infection: final report. Portland (OR): Oregon Health \& Science University; May 2007. Available at: http://www.ncbi.nlm. nih.gov/books/NBK10662/. Accessed April 25, 2018.

10. U.S. Food and Drug Administration. FDA approves Victrelis for hepatitis C. Press release. May 13, 2011. Updated April 10, 2014. Available at: https://wayback.archive-it.org/7993/20170112024030/http://www.fda.gov/ NewsEvents/Newsroom/PressAnnouncements/ucm255390.htm. Accessed April 25, 2018

11. Cox E. Incivek (telaprevir) approval letter. U.S. Food and Drug Administration. May 23, 2011. Available at: http://www.accessdata.fda.gov/ drugsatfda_docs/appletter/2011/201917Origls000ltr.pdf. Accessed April 25, 2018

12. Kiser JJ, Flexner C. Direct-acting antiviral agents for hepatitis C virus infection. Annu Rev Pharmacol Toxicol. 2013;53:427-49.

13. World Health Organization. Guidelines for the screening, care and treatment of persons with hepatitis C infection. April 2014. Available at: http://www. who.int/hiv/pub/hepatitis/hepatitis-c-guidelines/en/. Accessed April 25, 2018.

14. Sovaldi (sofosbuvir) for oral use. Gilead Sciences. November 2017. Available at: http://www.gilead.com/ /media/Files/pdfs/medicines/liverdisease/sovaldi/sovaldi_pi.pdf. Accessed April 25, 2018.

15. Olysio (simeprevir) for oral use. Janssen Therapeutics. 2013. Revised November 2017. Available at: http://www.olysio.com/shared/product/olysio/ prescribing-information.pdf. Accessed April 25, 2018.

16. U.S. Food and Drug Administration. FDA approves first combination pill to treat hepatitis C. Press release. Updated October 10, 2014. Available at: https://wayback.archive-it.org/7993/20161022101303/http://www.fda.gov/ NewsEvents/Newsroom/PressAnnouncements/ucm418365.htm. Accessed April 25, 2018

17. U.S. Food and Drug Administration. FDA approves Viekira Pak to treat hepatitis C. Press release. Updated December 22, 2014. Available at: https://wayback.archive-it.org/7993/20170111160836/http://www.fda.gov/ NewsEvents/Newsroom/PressAnnouncements/ucm427530.htm. Accessed April 25, 2018

18. Barua S, Greenwald R, Grebely J, Dore GJ, Swan T, Taylor LE. Restrictions for Medicaid reimbursement of sofosbuvir for the treatment of hepatitis $C$ virus infection in the United States. Ann Intern Med. 2015;163(3):215-23.

19. Westbrook RH, Dusheiko G. Natural history of hepatitis C. J Hepatol. 2014;61(1 Suppl):S58-68.

20. American Association for the Study of Liver Diseases-Infectious Diseases Society of America. Recommendations for testing, managing, and treating hepatitis C. Updated September 21, 2017. Available at: http://www.hcvguidelines.org/. Accessed April 25, 2018

21. Aday LA, Andersen R. A framework for the study of access to medical care. Health Serv Res. 1974;9(3):208-20.
22. Andersen RM. Revisiting the behavioral model and access to medical care: does it matter? J Health Soc Behav. 1995;36(1):1-10.

23. Steiner JF, Prochazka AV. The assessment of refill compliance using pharmacy records: methods, validity, and applications. J Clin Epidemiol. 1997;50(1):105-16.

24. Deyo RA, Cherkin DC, Ciol MA. Adapting a clinical comorbidity index for use with ICD-9-CM administrative databases. J Clin Epidemiol. 1992;45(6):613-19.

25. Sofair AN, Barry V, Manos MM, et al. The epidemiology and clinical characteristics of patients with newly diagnosed alcohol-related liver disease: results from population-based surveillance. J Clin Gastroenterol. 2010:44(4):301-07.

26. Kim WR, Brown RS Jr, Terrault NA, El-Serag H. Burden of liver disease in the United States: summary of a workshop. Hepatology. 2002;36(1):227-42.

27. Skrepnek GH, Olvey EL, Sahai A. Econometric approaches in evaluating costs and outcomes within pharmacoeconomic analyses. Pharm Policy Law. 2012;14:105-22

28. Skrepnek GH. Regression methods in the empiric analysis of health care data. J Manag Care Pharm. 2005;11(3):240-51. Available at: https://www. jmcp.org/doi/10.18553/jmcp.2005.11.3.240

29. Mohammad RA, Bulloch MN, Chan J, et al. Provision of clinical pharmacist services for individuals with chronic hepatitis $C$ viral infection: Joint Opinion of the GI/Liver/Nutrition and Infectious Diseases Practice and Research Networks of the American College of Clinical Pharmacy. Pharmacotherapy. 2014;34(12):1341-54.

30. Lawitz E, Sulkowski MS, Ghalib R, et al. Simeprevir plus sofosbuvir, with or without ribavirin, to treat chronic infection with hepatitis $C$ virus genotype 1 in non-responders to pegylated interferon and ribavirin and treatment-naive patients: the COSMOS randomised study. Lancet. 2014;384(9956):1756-65

31. Lawitz E, Matusow G, DeJesus E, et al. Simeprevir plus sofosbuvir in patients with chronic hepatitis $C$ virus genotype 1 infection and cirrhosis: a phase 3 study (OPTIMIST-2). Hepatology. 2016;64(2):360-69.

32. Afdhal N, Reddy KR, Nelson DR, et al. Ledipasvir and sofosbuvir for previously treated HCV genotype 1 infection. N Engl J Med. 2014;370(16):1483-93.

33. Afdhal N, Zeuzem S, Kwo P, et al. Ledipasvir and sofosbuvir for untreated HCV genotype 1 infection. N Engl J Med. 2014;370(20):1889-98.

34. Poordad F, Hezode C, Trinh R, et al. ABT-450/r-ombitasvir and dasabuvir with ribavirin for hepatitis $C$ with cirrhosis. N Engl J Med. 2014;370(21):1973-82.

35. Shin HP, Burman B, Kozarek RA, et al. Real-world single-center experience with sofosbuvir-based regimens for the treatment of chronic hepatitis $C$ genotype 1 patients. Gut Liver. 2017;11(5):711-20.

36. Brown RS Jr, O'Leary JG, Reddy KR, et al. Interferon-free therapy for genotype 1 hepatitis $C$ in liver transplant recipients: real-world experience from the hepatitis $C$ therapeutic registry and research network. Liver Transpl. 2016;22(1):24-33.

37. Ioannou GN, Beste LA, Chang MF, et al. Effectiveness of sofosbuvir, ledipasvir/sofosbuvir, or paritaprevir/ritonavir/ombitasvir and dasabuvir regimens for treatment of patients with hepatitis $C$ in the Veterans Affairs National Health Care System. Gastroenterology. 2016;151(3):457-71.e5.

38. Backus LI, Belperio PS, Shahoumian TA, Loomis TP, Mole LA. Realworld effectiveness and predictors of sustained virological response with all-oral therapy in 21,242 hepatitis C genotype-1 patients. Antivir Ther. 2017;22(6):481-93.

39. Dalgard $O$, Weiland $O$, Noraberg $G$, et al. Sofosbuvir based treatment of chronic hepatitis $C$ genotype 3 infections - a Scandinavian real-life study. PLoS One. 2017;12(7):e0179764. 
40. Reddy KR, Lim JK, Kuo A, et al. All-oral direct-acting antiviral therapy in HCV-advanced liver disease is effective in real-world practice: observations through HCV-TARGET database. Aliment Pharmacol Ther. 2017:45(1):115-26.

41. Falade-Nwulia O, Suarez-Cuervo C, Nelson DR, Fried MW, Segal JB, Sulkowski MS. Oral direct-acting agent therapy for hepatitis $C$ virus infection: a systematic review. Ann Intern Med. 2017;166(9):637-48.

42. Majumdar A, Kitson MT, Roberts SK. Systematic review: current concepts and challenges for the direct-acting antiviral era in hepatitis $\mathrm{C}$ cirrhosis. Aliment Pharmacol Ther. 2016;43(12):1276-92.

43. Guarino M, Morisco F, Valvano MR, et al. Systematic review: interferonfree regimens for patients with HCV-related Child C cirrhosis. Aliment Pharmacol Ther. 2017;45(9):1193-1200.
44. Nelson DR, Cooper JN, Lalezari JP, et al. All-oral 12-week treatment with daclatasvir plus sofosbuvir in patients with hepatitis $C$ virus genotype 3 infection: ALLY-3 phase III study. Hepatology. 2015;61(4):1127-35.

45. Grebely J, Page K, Sacks-Davis R, et al. The effects of female sex, viral genotype, and IL28B genotype on spontaneous clearance of acute hepatitis C virus infection. Hepatology. 2014;59(1):109-20.

46. Tsui JI, Mirzazadeh A, Hahn JA, et al. The effects of alcohol on spontaneous clearance of acute hepatitis $C$ virus infection in females versus males. Drug Alcohol Depend. 2016;169:156-62. 


\begin{tabular}{|c|c|c|}
\hline \multicolumn{3}{|c|}{$\begin{array}{ll}\text { APPENDIX A } & \text { Inflation Methodology for } \\
& \text { Prescription Drugs and Medical } \\
& \text { Care Services }\end{array}$} \\
\hline & CPI & Inflation Factora \\
\hline \multicolumn{3}{|c|}{$\begin{array}{l}\text { Prescription drugs from medical care component of CPI, } \\
\text { seasonally adjusted }\end{array}$} \\
\hline June 2013 & 440.310 & 1.175 \\
\hline June 2014 & 458.147 & 1.129 \\
\hline June 2015 & 480.019 & 1.078 \\
\hline June 2016 & 498.366 & 1.038 \\
\hline June 2017 & 517.455 & 1.000 \\
\hline
\end{tabular}

Medical care services from medical care component of CPI, seasonally adjusted

\begin{tabular}{l|l|l}
\hline June 2013 & 453.204 & 1.116 \\
\hline June 2014 & 464.808 & 1.088 \\
\hline June 2015 & 475.381 & 1.064 \\
\hline June 2016 & 493.291 & 1.025 \\
\hline June 2017 & 505.661 & 1.000 \\
\hline
\end{tabular}

Source: Bureau of Labor Statistics. All urban consumers (current series) Consumer Price Index (http://www.bls.gov/data/).

anflation factor was calculated by dividing the June 2017 CPI by each preceding year's CPI.

CPI = Consumer Price Index

\begin{tabular}{l|l|l}
\hline \multicolumn{1}{c}{ APPENDIX B } & $\begin{array}{l}\text { International Classification } \\
\text { of Diseases Codes for Liver } \\
\text { Sequelae }\end{array}$ \\
\hline & \multicolumn{1}{|c}{ ICD-95-CM } & \multicolumn{1}{c}{ ICD-10-CMa } \\
\hline Alcoholic cirrhosis & 571.2 & K70.30 \\
\hline Chronic hepatitis & 571.4 & $\begin{array}{l}\text { K73.9, K73.0, } \\
\text { K75.4, K73.2, K73.8 }\end{array}$ \\
\hline Nonalcoholic cirrhosis & 571.5 & $\begin{array}{l}\text { K74.0, K74.60, } \\
\text { K74.69 }\end{array}$ \\
\hline Biliary cirrhosis & 571.6 & K74.3, K74.4, K74.5 \\
\hline Hepatocellular carcinoma & 155.0 & C22.0, C22.2, \\
\hline Esophageal varices & $456.0,456.1$, & $\begin{array}{l}\text { I85.01, I85.00, } \\
\text { I85.11, I85.10 }\end{array}$ \\
\hline Hepatic encephalopathy & $456.20,456.21$ & K72.90, K72.91 \\
\hline Portal hypertension & 572.2 & K76.6 \\
\hline Hepatorenal syndrome & 572.3 & K76.7 \\
\hline $\begin{array}{l}\text { Other sequelae of chronic liver } \\
\text { disease }\end{array}$ & 572.4 & K72.10, K72.90 \\
\hline Gastrointestinal bleed & 578.8 & K92.0, K92.1, K92.2 \\
\hline Ascites & $789.5,549.1$ & R18.0, R18.8 \\
\hline Liver transplant (CPT-4 code) & 996.82, V42.7 & T86.40, T86.41, \\
& & T86.42, Z94.4 \\
\hline
\end{tabular}

aSource: ICD-10-CM CMS General Equivalence Mappings.

CMS = Centers for Medicare E Medicaid Services; CPT-4=Current Procedural Terminology, 4th Edition; ICD-9-CM = International Classification of Diseases, Ninth Revision, Clinical Modification; ICD-10-CM = International Classification of Diseases, Tenth Revision, Clinical Modification. 